\title{
Model-free control of manipulator in task space containing mismatched uncertainty
}

\author{
Xiaohui Yang - Zhenghong Xu - Wenjie Zhang - Wei Zhang - Peter \\ Xiaoping Liu
}

Received: date / Accepted: date

\begin{abstract}
In this paper, a novel trajectory tracking control method for manipulator task space containing mismatched uncertainty is proposed. This paper considers the mismatched uncertainty that exists in the signal conversion between joint space and task space in the robotic arm system. The time delay estimation is employed to approximate the dynamic and kinematic parameters of the system to achieve model-free control. The time delay estimation errors is treated as part of the system disturbances and a disturbance observer based on an auxiliary control system capable of estimating both matched and mismatched disturbances is designed, which does not require additional design of adaptive laws to compensate for disturbances or upper bounds on the derivatives of disturbances. We transform the control error by a prescribed performance function to accomplish transient control of the error, and design a fast terminal sliding surface for the virtual control variables based on the backstepping design method to improve the convergence speed of the controller. Based on the Lyapunov criterion, the closedloop stability of the whole system is demonstrated. The
\end{abstract}

X. Yang $\cdot \mathrm{Z} . \mathrm{Xu}(\otimes) \cdot \mathrm{W}$. Zhang $\cdot$ W.Zhang

School of Information Engineering, Nanchang University,

Nanchang 330031, Jiangxi, China

E-mail: 0b1ivi0u4@gmail.com

X. Yang

E-mail: yangxiaohui@ncu.edu.cn

W. Zhang

E-mail: yujianhn@163.com

W.Zhang

E-mail: 2358553149@qq.com

P.X. Liu

Department of Systems and Computer Engineering, Carleton

University, Ottawa, ON Canada K1S 5B6

E-mail: xpliu@sce.carleton.ca feasibility and superiority of the method in this paper is demonstrated by numerical simulation compared with other control methods.

Keywords Prescribed performance control · Backstepping design method - Mismatched uncertainty · Fast terminal sliding surface $\cdot$ Model-free control

\section{Introduction}

In recent years, as robotic manipulators are more and more widely applied in daily life and industry $[1,2,3$, $4,5]$, the control accuracy of robots is required to be higher and higher. However, because of the complex dynamics and uncertainties of robotic arm systems, it is still challenging to achieve high precision trajectory tracking of robots. Researchers have developed many control methods to address this problem, such as PID control $[6,7]$ for practical applications and many advanced control techniques designed to enhance control performance, such as model predictive control(MPC) $[8,9]$, sliding mode control(SMC) $[10,11]$, adaptive control $[12,13]$.

Since it is difficult to obtain an accurate system model of a manipulator during operation, the estimation of dynamic and kinematic parameters in the manipulator system and the estimation and compensation of external disturbances is considered one of the main problems to be solved by researchers. Many researchers have focused on the study of adaptive or robust controllers to achieve the required performance. [14] proposed an adaptive backstepping nonsingular fast terminal sliding mode controller, which employs adaptive technology to approach the upper bound of disturbances and uncertainties. In [15], an adaptive motion controller is designed that requires neither complicated 
gain constraints nor upper/lower bounds on model parameters in the dynamics for controller design and analysis. [16] proposed a model-independent adaptive fractional high-order terminal sliding mode controller and utilized an adaptive tuning control to evaluate the uncertain dynamics of the system without relying on the prior knowledge of the upper bounds. The main disadvantage of these methods is the inability to accurately estimate uncertainties and disturbances, resulting in a slightly conservative controller design. [17] developed an adaptive bias RBF neural network control for a robotic manipulator, which improves the approximation accuracy and the robustness of the RBFNN controller for the dynamics with significant bias. In [18], an adaptive fuzzy integral sliding-mode control is developed for robust fault-tolerant control of robot manipulators, and a fuzzy logic system is introduced to approximate the unknown nonlinear terms that include the model uncertainty and fault components. Similar to the above methods $[19,20]$, techniques that employ fuzzy logic or neural networks to estimate uncertainty can provide accurate estimations of unknown variables. However, they take a long time for the estimates to converge to the actual state in weight training by approximation rules, which is not conducive to real-time applications. The lumped uncertainty can also be estimated and compensated by designing disturbance observers or extended state observers [21,22,23], and these methods also enable to obtain accurate estimates of the unknowns. But traditional observers require upper bounds on the uncertainty or uncertainty time derivatives, which are difficult to obtain. Therefore researchers have proposed many methods that combine adaptive with observers. [24] designed an adaptive super-twisting disturbance observer with an adaptive law to update the gain parameters, which can estimate the system disturbances in fixed time. In [25], an adaptive sliding mode disturbance observer is proposed to estimate and compensate the system uncertainty, and similarly, an adaptive update law is proposed to estimate the upper bound of the first-order derivative value of the uncertainty. As shown in the above study, the design of the observer adaptive gain is often complex in order to make the overall closed-loop system stabilized. In addition, timedelay estimation (TDE) is also an efficient way to deal with uncertainty $[26,27,28]$. It can achieve accurate estimation of uncertainty by a simple structure, but when the uncertainty changes drastically in a short period of time, significant errors are generated, so the time-delay estimation error must be taken into consideration. To this end, one of the objectives of this paper is to propose a more feasible and less restrictive method for dealing with system uncertainty.
Moreover, all the above studies consider only matched uncertainties, while the manipulator system also has mismatched uncertainties. The mismatched uncertainties represent the case where the uncertainty terms impact the part of the system with no control input. Since there is no direct control signal in the influenced state, the matched part of the system should be changed to produce an indirect control effort to eliminate the effect of the mismatch uncertainties. And it is known that the backstepping control design has a unique superiority when the disturbances or uncertainties are not satisfied by the matched conditions [29, 30]. Therefore, this paper is based on the backstepping design approach for the controller design. Meanwhile, almost all of the above studies only consider the stability and steady-state error of the system and do not study the transient performance. The prescribed performance control can limit the control error to the range of the prescribed function and effectively control the transient performance, which has been widely used in various systems since it was proposed $[31,32]$.

According to the above discussion, this paper proposes a novel task space-based robotic arm trajectory tracking control method that involves mismatched uncertainties. Different from [33,34,18], which takes the joint angle variations on the joint space as the desired signal, this paper directly adopts the required trajectory of the manipulator in the task space as the desired trajectory and considers the uncertainties in the signal transformation between the joint space and the task space. The uncertainties are mismatched and not directly affected by the control inputs. This paper is based on the framework of backstepping design to facilitate the handling of mismatched uncertainty, first transforming the control error through a prescribed function to limit the transient error to a given range. Then a fast terminal sliding surface is designed for the following virtual control variables to enhance the controller convergence speed. In $[35,36,37]$, they use the time-delay method to estimate the lumped uncertainty including disturbances. We consider that the disturbances may change drastically in a short period of time, causing large time-delay estimation errors, greatly reduced control accuracy and robustness. While the model parameters generally fluctuate less and can be effectively estimated by the time-delay method, so we estimate the dynamic and kinematic parameters with TDE. However, the time delay estimation error is still a problem that needs to be considered. In this paper, the time delay estimation error is combined into the disturbance term. And for system disturbances, unlike [14,18,24, 38 ], due to the presence of mismatched disturbances, we adopt a less restrictive disturbance observer, which 
does not require the design of complex adaptive gains to compensate for the upper bound of the disturbance or the derivative of the disturbance. In summary, the main characteristics of this paper are as follows:

(1): Considering the mismatched uncertainties in the signal transformation between joint space and task space in the robotic arm system, accurate tracking based on the desired trajectory in task space is achieved.

(2): The model-free control is achieved by approximating the kinematic and dynamic parameters in the system by time delay estimation. And a disturbance observer that can observe both matched and mismatched disturbances is designed for disturbances that combine the time delay estimation errors.

(3): Based on the framework of backstepping design, the prescribed performance function is combined with fast terminal sliding mode control to improve the controller convergence speed while regulating the transient performance of the system. The Lyapunov criterion demonstrates the closed-loop stability of the whole system.

The rest of this paper is organized as follows. In Section II, we describe the problem to be solved and introduce the controlled robotic arm system. In Section III, a model-free adaptive control method is designed for controlled systems containing mismatched disturbances, and its closed-loop stability is demonstrated. Our controller is applied to 3-degree of-freedom robotic manipulators, and simulation results are presented in Section IV. The research conclusions are given in Section $\mathrm{V}$.

\section{Problem formulation}

The controller designed in this paper is applied to a robotic arm system that contains disturbances in signaling between joint space and task space. According to $[39,40]$, the dynamic model of the n-links manipulator in the joint space can be expressed as:

$M(q) \ddot{q}+C(q, \dot{q}) \dot{q}+G(q)=\tau_{d}+\tau$

where $q=\left[q_{1}, q_{2}, \ldots, q_{n}\right]^{T} \in \Re^{n}$ is a vector of joint angular postion. $\dot{q}, \ddot{q} \in \Re^{n}$ are the velocity and acceleration vectors of joints, respectively. $M(q) \in \Re^{n x n}$ is a positive define inertia matrix. $C(q, \dot{q}) \in \Re^{n x n}$ denotes the centripetal and coriolis matrix, $G(q) \in \Re^{n}$ is the vector of gravitational torques, $\tau_{d} \in \Re^{n}$ expresses the external disturbance signal and $\tau \in \Re^{n}$ is the vector of control torques.

The kinematic and differential mapping between the joint space and the task space can be described as:

$p=f(q)+f_{d}, \quad \dot{p}=J \dot{q}+\dot{f}_{d}$ where $f_{d} \in \Re^{m}$ is the disturbance signal in the kinematic of the system. $m \leq n, p=\left[p_{1}, p_{2}, \ldots, p_{m}\right]^{T} \in \Re^{m}$ are the coordinates of the end-effector in the task space. $f: \Re^{n} \rightarrow \Re^{m}$ denotes the mapping of the joint space to the task space constituted by the kinematic equations. $J=\frac{\partial f}{\partial q}$ is the $m \times n$ Jacobian matrix. We assume that the end-effector is required to track the desired trajectory $p_{d}(t) \in \Re^{m}$, which is at least triply continuously differentiable. Let the task tracking error $e(t)=p-p_{d}$, the objective of this paper is to design finite time control algorithm that accomplish the following equation:

$\lim _{t \rightarrow \infty} e(t)=\lim _{t \rightarrow \infty} \dot{e}(t)=0$

Since kinematic redundancy is not the focus of our discussion in this paper, it is assumed that $m=n$.

Assumption 1: The disturbances $\tau_{d}$ and $f_{d}$ are bounded. $f_{d}$ is at least first-order derivable.

And the following relevant properties are satisfied [40]:

Property 1: The inertia matrix $M(q)$ is positive definite symmetric. And $\forall q \in \Re^{n}$ the following inequalities are satisfied:

$k_{m} \leq\|M(q)\| \leq k_{M},\|C(q, \dot{q})\| \leq k_{C},\|G(q)\| \leq k_{G}$

where $k_{m}, k_{M}, k_{C}$ and $k_{G}$ are positive constants.

Property 2: The inertia matrix $M(q)$ and the centripetal Coriolis matrix $C(q, \dot{q})$ satisfy that

$x^{T}\left[\frac{1}{2} \dot{M}(q)-C(q, \dot{q})\right] x=0, \forall x \in \Re^{n}$

Property 3: In the kinematic model, there exist positive constants $k_{J}$ such that the following inequalities hold ture:

$0 \leq\|J\| \leq k_{J}, \forall q \in \Re^{n}$

Introducing constant matrix $\bar{M}=\operatorname{diag}\left(\bar{m}_{1}, \bar{m}_{2}, \ldots, \bar{m}_{n}\right)$ and $\bar{J}=\operatorname{diag}\left(\bar{j}_{1}, \bar{j}_{2}, \ldots, \bar{j}_{n}\right)$, based on (1) and (2) we can rewrite the system as

$\left\{\underline{p}=\bar{J} \dot{q}+N_{1}+\dot{f}_{d}\right.$

$\left\{\bar{M} \ddot{q}+N_{2}=\tau+\tau_{d}\right.$

where $N_{1}, N_{2}$ represent the kinematic and kinetic parameter terms of the system, respectively. $N_{1}=$ $[J(q)-\bar{J}] \dot{q}, N_{2}=[M(q)-\bar{M}] \ddot{q}+C(q, \dot{q}) \dot{q}+G(q)$. Based on the above properties, we can know that $N_{1}$ and $N_{2}$ are bounded.

\section{Control of the robotic manipulator}

In this section we develop an novel controller for a manipulator system containing mismatched disturbances based on sliding mode control and prescribed performance function with the backstepping design technique. 


\subsection{Model-based controller}

In this part, based on the above kinematic and dynamical model of the robotic arm, we design a controller for the accurate model of the manipulator system to achieve the tracking of the desired trajectory in the framework of the backstepping method combined with sliding mode control and prescribed performance control. For uncertainties and disturbances, they will be estimated and compensated by time delay estimation and disturbance observer in the later section.

Let $x_{1}=p, x_{2}=\dot{q}, x_{1}, x_{2} \in \Re^{n}$, then system (4) can be rewrited as:

$\left\{\begin{array}{l}\dot{x}_{1}=\bar{J} x_{2}+N_{1}+d_{1} \\ \dot{x}_{2}=\bar{M}^{-1} \tau-\bar{M}^{-1} N_{2}+d_{2}\end{array}\right.$

where $d_{1}=\dot{f}_{d}, d_{2}=\bar{M}^{-1} \tau_{d}, d_{1}$ and $d_{2}$ are the mismatched and matched disturbances of the system, respectively. And

$$
\begin{aligned}
\dot{e}(t) & =\dot{x}_{1}-\dot{p}_{d} \\
& =\bar{J} x_{2}+N_{1}+d_{1}-\dot{p}_{d}
\end{aligned}
$$

To improve the transient and steady-state performance of the system, the trajectory tracking errors are required to satisfy the following inequality

$-\delta_{i} v(t)<e_{i}<\delta_{i} v(t)$

where $e_{i}(i=1,2, \ldots, n)$ denotes the $i$ th element of $e(t)$, and $0<\delta_{i} \leq 1$ is a designed constant.

Then we introduce the following finite-time prescribed performance function:

$v(t)= \begin{cases}\left(v_{0}-\frac{t}{T_{f}}\right) e^{\left(1-\frac{T_{f}}{T_{f}-t}\right)}+v_{T_{f}}, t \in\left[0, T_{f}\right) \\ v_{T_{f}}, t \in\left[T_{f},+\infty\right)\end{cases}$

where $v_{0} \geq 1$ and $v_{T_{f}}>0$ are the design parameters. From(), performance function $v(t)$ is positive smooth exponential decreasing with $\lim _{t \rightarrow T_{f}} v(t)=v_{T_{f}}>0$ and $v(t)=v_{T_{f}}$ for any $t \geq T_{f}$.

To achieve prescribed performance control, we introduce the following error transformation method to convert the constrained error in (6) into an equivalent unconstrained error $\varepsilon(t)$, abbreviated as $\varepsilon$.

$Q\left(\varepsilon_{i}\right)=\delta_{i} \frac{e^{\varepsilon_{i}}-e^{-\varepsilon_{i}}}{e^{\varepsilon_{i}}+e^{-\varepsilon_{i}}}=\frac{e_{i}(t)}{v(t)}$

Property 4: $Q\left(\varepsilon_{i}\right)$ is a smooth and strictly increasing function, $\lim _{\varepsilon_{i} \rightarrow-\infty} Q\left(\varepsilon_{i}\right)=-\delta_{i}, \lim _{\varepsilon_{i} \rightarrow+\infty} Q\left(\varepsilon_{i}\right)=$ $\delta_{i}$.
From the definition of $Q\left(\varepsilon_{i}\right)$, we obtain

$\varepsilon_{i}=Q^{-1}\left(\frac{e_{i}(t)}{v(t)}\right)=\frac{1}{2} \ln \frac{\delta_{i} v(t)+e_{i}(t)}{\delta_{i} v(t)-e_{i}(t)}$

and

$\frac{\partial Q\left(\varepsilon_{i}\right)}{\partial \varepsilon_{i}}=\delta_{i}-\delta_{i}\left(\frac{e^{\varepsilon_{i}}-e^{-\varepsilon_{i}}}{e^{\varepsilon_{i}}+e^{-\varepsilon_{i}}}\right)^{2}$

We can get $e_{i}(t)=v(t) Q\left(\varepsilon_{i}\right)$, then we know

$\dot{e}_{i}(t)=\dot{v}(t) Q\left(\varepsilon_{i}\right)+v(t) \frac{\partial Q\left(\varepsilon_{i}\right)}{\partial \varepsilon_{i}} \dot{\varepsilon}_{i}$

According to $e(t)=p-p_{d}$, we can derive

$$
\begin{aligned}
\dot{\varepsilon}_{i} & =\frac{\dot{e}_{i}(t)-\dot{v}(t) Q\left(\varepsilon_{i}\right)}{v(t) \frac{\partial Q\left(\varepsilon_{i}\right)}{\partial \varepsilon_{i}}} \\
& =\frac{\dot{e}_{i}(t)}{v(t) \frac{\partial Q\left(\varepsilon_{i}\right)}{\partial \varepsilon_{i}}}-\frac{\dot{v}(t) Q\left(\varepsilon_{i}\right)}{v(t) \frac{\partial Q\left(\varepsilon_{i}\right)}{\partial \varepsilon_{i}}} \\
& =\Psi\left(\varepsilon_{i}, t\right) \dot{e}_{i}(t)+\phi\left(\varepsilon_{i}, t\right)
\end{aligned}
$$

with

$$
\begin{aligned}
\Psi\left(\varepsilon_{i}, t\right) & =\frac{1}{v(t) \frac{\partial Q\left(\varepsilon_{i}\right)}{\partial \varepsilon_{i}}} \\
\phi\left(\varepsilon_{i}, t\right) & =-\frac{\dot{v}(t) Q\left(\varepsilon_{i}\right)}{v(t) \frac{\partial Q\left(\varepsilon_{i}\right)}{\partial \varepsilon_{i}}}
\end{aligned}
$$

Applying the backstepping design method, the trajectory tracking controller designed for the system in (5) is as follows.

Step 1 : To facilitate the subsequent derivation, we first define two auxiliary variables as $z_{1}=\varepsilon$, $z_{2}=x_{2}-\alpha$, where $\alpha$ is a vector of virtual control input. We design $\alpha$ as

$\alpha=\frac{1}{\bar{J}}\left[-N_{1}+\dot{p}_{d}-\hat{d}_{1}-k_{1} z_{1}-\frac{\phi(\varepsilon, t)}{\Psi(\varepsilon, t)}\right]$

where $\hat{d}_{1}$ is the estimation of $d_{1}$, obtained from the observer designed later.

Consider the Lyapunov function as $V_{1}=\frac{1}{2} z_{1}^{T} z_{1}$, then according to (13) and (14), we can obtain the time derivative of $V_{1}$

$$
\begin{aligned}
\dot{V}_{1} & =z_{1}^{T}\left[\Psi(\varepsilon, t)\left(\bar{J} x_{2}+N_{1}+d_{1}-\dot{p}_{d}\right)+\phi(\varepsilon, t)\right] \\
& =z_{1}^{T}\left[\Psi(\varepsilon, t)\left(\bar{J}\left(z_{2}+\alpha\right)+N_{1}+d_{1}-\dot{p}_{d}\right)+\phi(\varepsilon, t)\right] \\
& =-k_{1} \Psi(\varepsilon, t) z_{1}^{T} z_{1}+\Psi(\varepsilon, t) \bar{J} z_{1}^{T} z_{2}+\Psi(\varepsilon, t) z_{1}^{T} \tilde{d}_{1}
\end{aligned}
$$

where $\tilde{d}_{1}=d_{1}-\hat{d}_{1}$ is the vector of estimation errors. By the definition of $\Psi\left(\varepsilon_{i}, t\right)$ we know that $\Psi\left(\varepsilon_{i}, t\right)>0$ and is bounded. 
Step 2 : To enhance the robustness of the controller and improve the convergence speed, we introduce the sliding surface. Define a switching function as

$s=z_{2}+c_{1} \operatorname{sign}^{a_{1}}\left(z_{1}\right)+c_{2} \operatorname{sign}^{a_{2}}\left(z_{1}\right)$

where $\quad c_{1}=\operatorname{diag}\left(c_{11}, c_{12}, \ldots, c_{1 n}\right)$, $c_{2}=\operatorname{diag}\left(c_{21}, c_{22}, \ldots, c_{2 n}\right)$ are the gain coefficient matrices; $0<a_{1}<1,1<a_{2}<2$. Choose a Lyapunov candidate function as $V_{2}=V_{1}+\frac{1}{2} s^{T} s$. Applying (16) and according to the definitions of $z_{1}, z_{2}$, it leads to

$$
\begin{aligned}
\dot{V}_{2}= & \dot{V}_{1}+s^{T}\left(\dot{z}_{2}+c_{1} a_{1} \operatorname{sign}^{a_{1}-1} z_{1} \dot{z}_{1}+c_{2} a_{2} \operatorname{sign}^{a_{2}-1} z_{1} \dot{z}_{1}\right) \\
= & \dot{V}_{1}+s^{T}\left[\Xi\left(z_{1}\right)(\Psi(\varepsilon, t) \dot{e}(t)+\phi(\varepsilon, t))+\dot{x}_{2}-\dot{\alpha}\right] \\
= & -k_{1} \Psi(\varepsilon, t) z_{1}^{T} z_{1}+\Psi(\varepsilon, t) \bar{J} z_{1}^{T} z_{2}+\Psi(\varepsilon, t) z_{1}^{T} \tilde{d}_{1} \\
& +s^{T}\left[\Xi\left(z_{1}\right)(\Psi(\varepsilon, t) \dot{e}(t)+\phi(\varepsilon, t))\right. \\
& \left.\left.+\bar{M}^{-1} \tau-\bar{M}^{-1} N_{2}+d_{2}-\dot{\alpha}\right)\right]
\end{aligned}
$$

where $\Xi\left(z_{1}\right)=c_{1} a_{1} \operatorname{sign}^{a_{1}-1} z_{1}+c_{2} a_{2} \operatorname{sign}^{a_{2}-1} z_{1}$.

Then we can design the controller $\tau$ as

$$
\begin{aligned}
\tau= & \bar{M}\left[-\Xi\left(z_{1}\right)(\Psi(\varepsilon, t) \dot{e}(t)-\phi(\varepsilon, t))+\bar{M}^{-1} N_{2}\right. \\
& -\hat{d}_{2}-\Psi(\varepsilon, t) \bar{J} z_{1}+\dot{\alpha}-h(s+\beta \operatorname{sign}(s)]
\end{aligned}
$$

where $h, \beta$ are positive constant matrix and $\hat{d}_{2}$ is the estimation of disturbance term $d_{2}$, it is designed in the later content.

Remark 1: The proposed controller requires the position and velocity signals of the end-effector and the angular velocity signals of the joints. However, the velocity of the end-effector and the angular velocity of the joints cannot be available directly, while the position of the end-effector is measurable and the joint position can be obtained directly from the encoder. Therefore, in order to estimate the joints angular velocities and end-effector velocities, we adopt first-order exact differential [41].

$$
\begin{aligned}
& \dot{\omega}_{0}=\kappa_{q}, \dot{\zeta}_{0}=\kappa_{p} \\
& \kappa_{q}=-\lambda_{0}\left|\omega_{0}-q\right|^{1 / 2} \operatorname{sign}\left(\omega_{0}-q\right)+\omega_{1} \\
& \kappa_{p}=-\gamma_{0}\left|\zeta_{0}-q\right|^{1 / 2} \operatorname{sign}\left(\zeta_{0}-q\right)+\zeta_{1} \\
& \dot{\omega}_{1}=-\lambda_{1} \operatorname{sign}\left(\omega_{1}-\kappa\right) \\
& \dot{\zeta}_{1}=-\gamma_{1} \operatorname{sign}(\zeta 1-\kappa)
\end{aligned}
$$

where $\lambda_{0}, \lambda_{1}, \gamma_{0}$ and $\gamma_{1}$ are positive constants. Then we can obtain $\omega_{0}=q, \omega_{1}=\dot{q}=x_{2}, \zeta_{0}=p, \zeta_{1}=\dot{p}$.

\subsection{Time delay estimation}

It is known that the accurate model of the manipulator is difficult to obtain, and there is uncertainty in the manipulator model, which means that $N_{1}$ and $N_{2}$ in (5) are unknown. To effectively solve this problem, we apply the TDE to estimate the unknown kinematic and dynamic parameters, $\hat{N}_{1}, \hat{N}_{2}$ are the estimation of $N_{1}$, $N_{2}$. A simple struture as

$\hat{N}_{1}(t) \cong N_{1}(t-T)$

$\hat{N}_{2}(t) \cong N_{2}(t-T)$

where $T$ stands for a very short time delay constant and it is generally set to a sampling time.

Then the kinematic and dynamic equations at time $(t-T)$ can be rewritten as

$$
\left\{\begin{array}{l}
\dot{x}_{1}(t-T)=\bar{J} x_{2}(t-T)+N_{1}(t-T)+d_{1}(t-T) \\
\dot{x}_{2}(t-T)=\bar{M}^{-1} \tau(t-T)-\bar{M}^{-1} N_{2}(t-T)+d_{2}(t-T)
\end{array}\right.
$$

where $d_{1}, d_{2}$ are unknown disturbance term. We cannot directly obtain their exact values at the last sampling moment, so we design the disturbance observer to get their estimated values $\hat{d}_{1}, \hat{d}_{2}$.

Substituting $\hat{d}_{1}, \hat{d}_{2}$ and combining (20) with (21), we get

$\hat{N}_{1}(t)=\bar{J} x_{2}(t-T)-\dot{x}_{1}(t-T)+\hat{d}_{1}(t-T)$
$\hat{N}_{2}(t)=\tau(t-T)-\bar{M} \dot{x}_{2}(t-T)+\hat{d}_{2}(t-T)$

From (22), we need the values of $\tau(t-T)$ and $\dot{x}_{2}(t-T)$, where $\tau(t-T)$ is the value of the control signal $\tau$ at the previous moment, which is available, and $\dot{x}_{2}(t-T)$ can be obtained by the exact differentiator in the previous section, so the TDE can effectively achieve model-free control.

The bounded TDE error vector is defined as follows:

$\theta_{1}=\left(N_{1}-\hat{N}_{1}\right)$
$\theta_{2}=\left(N_{2}-\hat{N}_{2}\right)$

It is known that the TDE error $\theta_{1}$ and $\theta_{2}$ is bounded, determined by $[42,43]$.

To guarantee that the time delay estimation error is bounded, the following inequalities must be satisfied

$$
\begin{aligned}
\left\|I-J^{-1} \bar{J}\right\| & <1 \\
\left\|I-M^{-1}(q) \bar{M}\right\| & <1
\end{aligned}
$$

Assume $\varpi_{i}$ is the $i$ th eigenvalue of $M(q)$, according to Property 1, $\varpi_{i}$ is bounded. Let $\eta_{i}$ is the $i$ th eigenvalue of matrix $\left(I-M^{-1}(q) \bar{M}\right)$, then $\eta_{i}=1-\bar{m}_{i} / \varpi_{i}$. 
In order to satisfy the inequality $\left\|I-M^{-1}(q) \bar{M}\right\|<1$, $-1<\eta_{i}<1$ is a sufficient condition, i.e., $-1<$ $1-\bar{m}_{i} / \varpi_{i}<1$ allows the required inequality to hold. Then we only need to choose $0<\bar{m}_{i}<2 \varpi_{i}$. And since $\varpi_{i}$ is bounded, a reasonable matrix $\bar{M}$ can be found to make the above inequality hold. Similarly, inequality $\left\|I-M^{-1}(q) \bar{M}\right\|<1$ can be accomplished by selecting appropriate $\bar{J}$.

To deal with the time delay estimation errors, we treat they as part of the system disturbances. Then $d_{1}$ and $d_{2}$ can be rewritten as

$d_{1}=\theta_{1}+\dot{f}_{d}$

$d_{2}=\bar{M}^{-1}\left(\tau_{d}-\theta_{2}\right)$

Then system (5) can be formulated as:

$\left\{\begin{array}{l}\dot{x}_{1}=\bar{J} x_{2}+\hat{N}_{1}+d_{1} \\ \dot{x}_{2}=\bar{M}^{-1} \tau-\bar{M}^{-1} \hat{N}_{2}+d_{2}\end{array}\right.$

\subsection{Disturbance estimation design}

To facilitate the design of the estimator, we first convert the system (26) to the following form

$\dot{X}=-H X+G(X)+B \tau+\Delta$

where $X=\left[x_{1}, x_{2}\right]^{T}, H=\operatorname{diag}\left(h_{1}, h_{2}\right), h_{1}=$ $\operatorname{diag}\left(h_{11}, h_{12}, \ldots, h_{1 n}\right), \quad h_{2}=\operatorname{diag}\left(h_{21}, h_{22}, \ldots, h_{2 n}\right)$, $G(X)=\left[\begin{array}{c}h_{1} x_{1}+\bar{J} x_{2}+\hat{N}_{1} \\ h_{2} x_{2}-\bar{M}^{-1} \hat{N}_{2}\end{array}\right], B=\left[0, \bar{M}^{-1}\right]^{T}, \Delta=$ $\left[d_{1}, d_{2}\right]^{T}$.

To construct an observer capable of estimating both matched and mismatched disturbances, we first design the following auxiliary control system

$\dot{X}_{a}=-H X_{a}+G(X)+B \tau$

As shown above, $H, G(X), B$ and $\tau$ are known variables, so the state $X_{a}$ of the auxiliary system is measurable.

Define the errors between $X$ and $X_{a}$ as $X_{e}$, according to (27) and (28), we can obtain

$\dot{X}_{e}=-H X_{e}+\Delta$

We consider $\Delta$ as an unknown input, then (29) can be treated as a linear system.

Let $\hat{X}_{e}$ represent the estimated value of $X_{e}$ and design the following structure for $\hat{X}_{e}$

$\dot{\hat{X}}_{e}=-L \hat{X}_{e}+\dot{X}_{e}+L X_{e}$

where $L \in \Re^{2 n \times 2 n}$ is a positive-definite and diagonal matrix. $L=\operatorname{diag}\left(L_{1}, L_{2}\right), L_{1}=\operatorname{diag}\left(l_{11}, l_{12}, \ldots, l_{1 n}\right)$, $L_{2}=\operatorname{diag}\left(l_{21}, l_{22}, \ldots, l_{2 n}\right)$.
According to (29), we can obtain an estimation of the disturbance $\Delta$ of the following form

$\hat{\Delta}=H \hat{X}_{e}+\dot{X}_{e}$

Let the error between the estimation of $\hat{X}_{e}$ and $X_{e}$ as $\tilde{X}_{e}=X_{e}-\hat{X}_{e}$. From (29) and (30) we can get

$$
\begin{aligned}
\dot{\tilde{X}}_{e} & =\Delta-H X_{e}-\left(-L \hat{X}_{e}+\dot{X}_{e}+L X_{e}\right) \\
& =-L \tilde{X}_{e}
\end{aligned}
$$

Subtracting $\hat{\Delta}$ from $\Delta$, based on (31) we can derive the disturbance estimation error that satisfies

$$
\begin{aligned}
\tilde{\Delta} & =\Delta-\hat{\Delta} \\
& =\Delta-\left(H \hat{X}_{e}+\dot{X}_{e}\right) \\
& =\Delta-\left(H \hat{X}_{e}-H X_{e}+\Delta\right) \\
& =H \tilde{X}_{e}
\end{aligned}
$$

Remark 2: In contrast to existing ESO, DO, etc $[18,24,38]$, the observer employed in this paper does not require any conditions or assumptions about disturbances. Although in Assumption 1, we assume that $f_{d}$ and $\tau_{d}$ are bounded and $f_{d}$ is derivable. However, the bounds of the disturbance are unknown, we don't need their upper bound values. And if the disturbance is infinite, the system is not controllable. The assumption that $f_{d}$ is derivable is to transform the system into the equation of state, not a condition required by this disturbance observer. Moreover the closed-loop stability containing the observation error is demonstrated in the next section. Thus the disturbance observer in this paper can effectively handle both matched and mismatched disturbances.

\subsection{Proof of closed-loop system}

Substituting the estimation results of the above disturbance estimator into the virtual control variable $\alpha$ and control law $\tau$.

$$
\begin{aligned}
\alpha= & \frac{1}{\bar{J}}\left[-\hat{N}_{1}+\dot{p}_{d}-\hat{d}_{1}-k_{1} z_{1}-\frac{\phi(\varepsilon, t)}{\Psi(\varepsilon, t)}\right] \\
\tau=\bar{M} & {\left[-\Xi\left(z_{1}\right)(\Psi(\varepsilon, t) \dot{e}(t)-\phi(\varepsilon, t))+\bar{M}^{-1} \hat{N}_{2}\right.} \\
& -\hat{d}_{2}-\Psi(\varepsilon, t) \bar{J} z_{1}+\dot{\alpha}-h(s+\beta \operatorname{sign}(s)]
\end{aligned}
$$




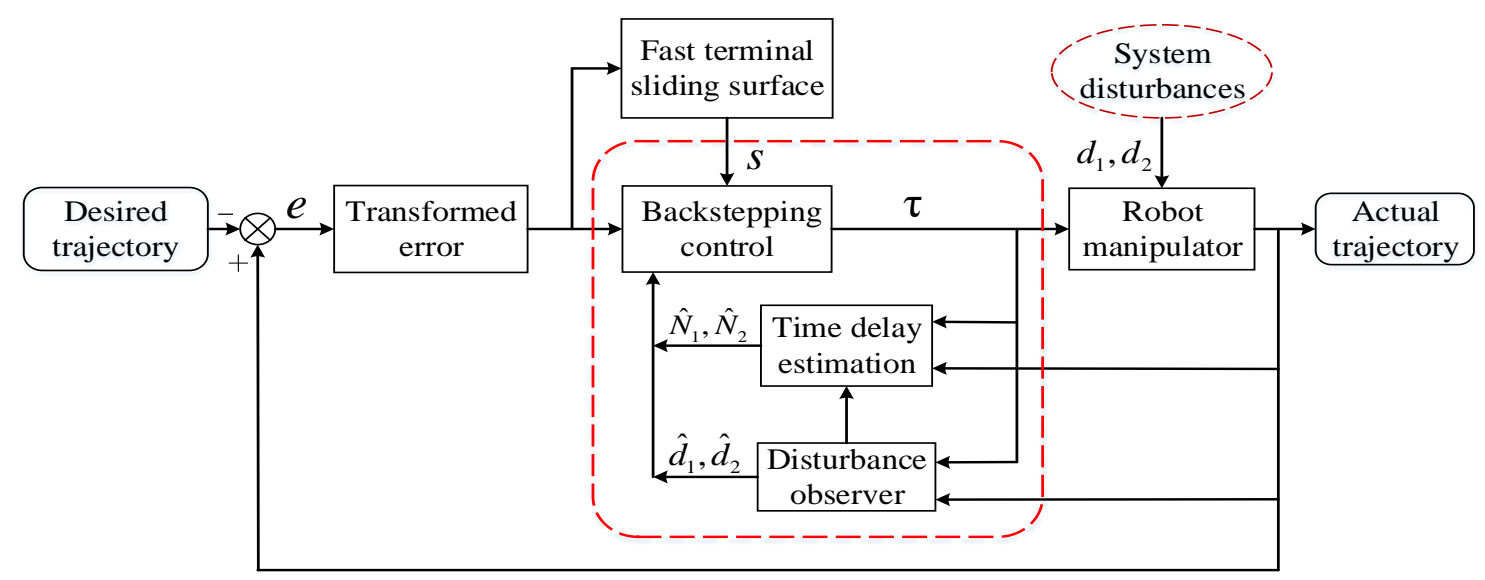

Fig. 1: Sketch of the proposed ABIFTSMC based on ESO

Choose a Lyapunov function as $V_{3}=V_{2}+\frac{1}{2} \tilde{\Delta}^{T} \tilde{\Delta}$. It can be deduced from (14) and (18) that

$$
\begin{aligned}
\dot{V}_{3}= & \dot{V}_{2}+\tilde{\Delta}^{T} \dot{\tilde{\Delta}} \\
\dot{V}_{3}= & -k_{1} \Psi(\varepsilon, t) z_{1}^{T} z_{1}-h s^{T} s-h \beta \sum_{i=1}^{n}\left|s_{i}\right| \\
& -\Psi(\varepsilon, t) \bar{J} \sum_{i=1}^{n} c_{1, i}\left|z_{1, i}\right|^{a_{1}+1}-\Psi(\varepsilon, t) \bar{J} \sum_{i=1}^{n} c_{2, i}\left|z_{1, i}\right|^{a_{2}+1} \\
& +\Psi(\varepsilon, t) z_{1}^{T} \tilde{d}_{1}+s^{T} \tilde{d}_{2}+\tilde{\Delta}^{T} \dot{\tilde{\Delta}}
\end{aligned}
$$

Based on (32) and (33), we can obtain

$$
\begin{aligned}
\tilde{\Delta}^{T} \dot{\tilde{\Delta}} & =\tilde{\Delta}^{T} H \dot{\tilde{X}}_{e} \\
& =-\tilde{\Delta}^{T} H L \tilde{X}_{e} \\
& =-\tilde{\Delta}^{T} L \tilde{\Delta} \\
& =-\tilde{d}_{1}^{T} L_{1} \tilde{d}_{1}-\tilde{d}_{2}^{T} L_{2} \tilde{d}_{2}
\end{aligned}
$$

Then the following inequality can be given

$$
\tilde{\Delta}^{T} \dot{\tilde{\Delta}} \leq-\lambda_{\min }\left(L_{1}\right)\left\|\tilde{d}_{1}\right\|^{2}-\lambda_{\min }\left(L_{2}\right)\left\|\tilde{d}_{2}\right\|^{2}
$$

It is well known that the following inequalities hold

$$
\begin{aligned}
z_{1}^{T} \tilde{d}_{1} & \leq \frac{1}{r_{1}}\left\|z_{1}\right\|^{2}+r_{1}\left\|\tilde{d}_{1}\right\|^{2} \\
s^{T} \tilde{d}_{2} & \leq \frac{1}{r_{2}}\|s\|^{2}+r_{2}\left\|\tilde{d}_{2}\right\|^{2}
\end{aligned}
$$

Therefore, based on the above inequality, it is deduced that

$$
\begin{aligned}
\dot{V}_{3} \leq & -k_{1} \Psi(\varepsilon, t) z_{1}^{T} z_{1}-h s^{T} s \\
& +\frac{1}{r_{1}} \Psi(\varepsilon, t)\left\|z_{1}\right\|^{2}+r_{1} \Psi(\varepsilon, t)\left\|\tilde{d}_{1}\right\|^{2}+\frac{1}{r_{2}}\|s\|^{2}+r_{2}\left\|\tilde{d}_{2}\right\|^{2} \\
& -\lambda_{\min }\left(L_{1}\right)\left\|\tilde{d}_{1}\right\|^{2}-\lambda_{\min }\left(L_{2}\right)\left\|\tilde{d}_{2}\right\|^{2} \\
\leq & -\Psi(\varepsilon, t)\left(k_{1}-\frac{1}{r_{1}}\right)\left\|z_{1}\right\|^{2}-\left(h-\frac{1}{r_{2}}\right)\|s\|^{2} \\
& -\left(\lambda_{\min }\left(L_{1}\right)-r_{1} \Psi(\varepsilon, t)\right)\left\|\tilde{d}_{1}\right\|^{2} \\
& -\left(\lambda_{\min }\left(L_{2}\right)-r_{2}\right)\left\|\tilde{d}_{2}\right\|^{2}
\end{aligned}
$$

To achieve closed-loop system stability, it is only necessary to choose $k_{1}, h, L_{1}$ and $L_{2}$ to satisfy the following inequalities

$$
\begin{aligned}
& k_{1}-\frac{1}{r_{1}}>0, h-\frac{1}{r_{2}}>0 \\
& \lambda_{\min }\left(L_{1}\right)-r_{1} \Psi(\varepsilon, t)>0, \lambda_{\min }\left(L_{2}\right)-r_{2}>0
\end{aligned}
$$

where $r_{1}, r_{2} \in \Re$ are positive scalars and determined by the designer.

Remark 3: From the definition of $\Psi(\varepsilon, t)$, it is clear that $\Psi(\varepsilon, t)$ i 0 and bounded. And $r_{1}$ and $r_{2}$ are chosen by the designer, so the design parameters are feasible to satisfy the above inequalities. The stability of the closed-loop system can be achieved by designing the appropriate parameters.

\section{Numerical simulation}

In this section, the performance of the proposed approach is verified by numerical simulation of the threelink anthropomorphic robotic arm shown in Fig.2. $q_{1}, q_{2}$ 


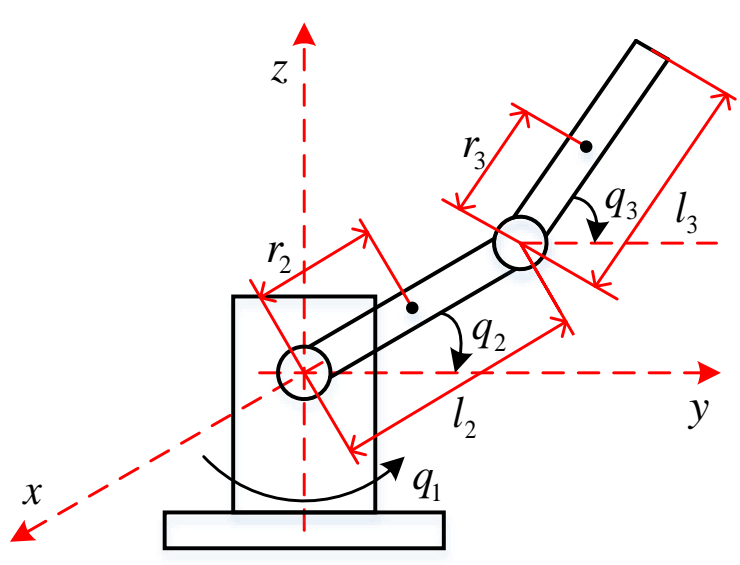

Fig. 2: The architecture of two-link robotic manipulators

and $q_{3}$ represent the angle positions of joints, and $\dot{q}_{1}, \dot{q}_{2}$ and $\dot{q}_{2}$ are the angular velocities of joints. The kinematic equations of the three-joint manipulator are as follows

$$
\left\{\begin{array}{l}
\left.x=\cos q_{1}\left(l_{2} \cos q_{2}\right)+l_{3} \cos \left(q_{2}+\theta_{3}\right)\right) \\
\left.y=\sin q_{1}\left(l_{2} \cos q_{2}\right)+l_{3} \cos \left(q_{2}+q_{3}\right)\right) \\
z=l_{2} \sin q_{2}+l_{3} \sin \left(q_{2}+q_{3}\right)
\end{array}\right.
$$

where $(x, y, z)$ is the end-effector position of the manipulator.

The dynamic of the manipulator in (1) by Lagrangian equation is represented as

$$
\begin{aligned}
& M(q)=\left[\begin{array}{lll}
m_{11} & m_{12} & m_{13} \\
m_{21} & m_{22} & m_{23} \\
m_{31} & m_{32} & m_{33}
\end{array}\right], G(q)=\left[\begin{array}{l}
g_{1} \\
g_{2} \\
g_{3}
\end{array}\right], \\
& C(q, \dot{q})=\left[\begin{array}{lll}
c_{11} & c_{12} & c_{13} \\
c_{21} & c_{22} & c_{23} \\
c_{31} & c_{32} & c_{33}
\end{array}\right] .
\end{aligned}
$$$$
m_{11}=I_{1}+a_{1} \cos ^{2} q_{2}+a_{2} \cos \left(q_{2}+q_{3}\right)+
$$$$
2 a_{3} \cos q_{2} \cos \left(q_{2}+q_{3}\right)
$$$$
m_{12}=m_{21}=m_{13}=m_{31}=0, m_{33}=I_{3}+a_{2} \text {, }
$$$$
m_{22}=I_{2}+a_{1}+a_{2}+2 a_{3} \cos q_{3} \text {, }
$$$$
m_{23}=m_{32}=a_{2}+a_{3} \cos q_{3} \text {, }
$$

Table 1: Physical parameters of the two-link robotic manipulator.

\begin{tabular}{lll}
\hline Symbol & \multicolumn{1}{c}{ Definition } & \multicolumn{1}{c}{ Value } \\
\hline$m_{1}$ & mass of link 1 & $10 \mathrm{~kg}$ \\
$m_{2}$ & mass of link 2 & $8 \mathrm{~kg}$ \\
$l_{2}$ & Length of link 2 & $0.5 \mathrm{~m}$ \\
$r_{2}$ & Length of the center of mass of link 1 & $0.254 \mathrm{~m}$ \\
$l_{3}$ & Length of link 3 & $0.4 \mathrm{~m}$ \\
$r_{3}$ & Length of the center of mass of link 2 & $0.205 \mathrm{~m}$ \\
$g$ & Gravitational constant & $9.81 \mathrm{~m} / \mathrm{s}$ \\
\hline
\end{tabular}

$c_{11}=-\frac{1}{2} a_{1} \dot{q}_{2} \sin \left(2 q_{2}\right)-a_{3} \dot{q}_{2} \sin \left(2 q_{2}+q_{3}\right)-$

$$
\frac{1}{2} a_{2}\left(\dot{q}_{2}+\dot{q}_{3}\right) \sin \left(2 q_{2}+2 q_{3}\right)-
$$$$
a_{3} \dot{q}_{3} \cos q_{2} \sin \left(q_{2}+q_{3}\right)
$$

$c_{12}=-\frac{1}{2} a_{1} \dot{q}_{1} \sin \left(2 q_{2}\right)-a_{3} \dot{q}_{1} \sin \left(2 q_{2}+q_{3}\right)-$

$$
\frac{1}{2} a_{2} \dot{q}_{1} \sin \left(2 q_{2}+2 q_{3}\right),
$$

$c_{13}=-a_{3} \dot{q}_{1} \cos q_{2} \sin \left(q_{2}+q_{3}\right)-$

$$
\frac{1}{2} a_{1} \dot{q}_{1} \sin \left(2 q_{2}+2 q_{3}\right) \text {, }
$$

$c_{21}=-c_{12}, c_{31}=-c_{13}, c_{33}=0$

$c_{23}=-a_{3}\left(\dot{q}_{2}+\dot{q}_{3}\right) \sin q_{3}$,

$c_{32}=-a_{3} \dot{q}_{2} \sin q_{3}, c_{22}=-a_{3} \dot{q}_{3} \sin q_{3}$,

$g_{1}=0, g_{2}=b_{1} \cos q_{2}+b_{2} \cos \left(q_{2}+q_{3}\right)$,

$g_{3}=b_{2} \cos \left(q_{2}+q_{3}\right)$.

where $a_{1}=m_{2} r_{2}^{2}+m_{3} l_{2}^{2}, a_{2}=m_{3} r_{3}^{2}, a_{3}=m_{3} r_{3} l_{3}$, $b_{1}=\left(m_{2} r_{2}+m_{3} l_{2}\right) g, b_{2}=m_{3} r_{3} g$.

Table 1 lists the basic parameters of the robotic arm for numerical simulation

The initial values of the system are selected as $p_{0}=[0.373,0.384,0.4987]^{T}, \dot{q}_{0}=[0,0,0]^{T}$, respectively The desired trajectories are selected as

$\left\{\begin{array}{l}x=0.3+0.1 \times \cos (t) \\ y=0.3+0.1 \times \sin (t) \\ z=0.2-0.033 \times t\end{array}\right.$

To check the robustness of the system, we add the following disturbances to the system

$$
\begin{aligned}
& d_{1}=\left[\begin{array}{l}
0.5 q_{1}+\sin \left(3 q_{1}\right)+\sin (t) \\
1.3 q_{2}-1.8 \sin \left(2 * q_{2}\right)+0.8 \sin (t) \\
-1.8 q_{3}+2 \sin \left(3 * q_{3}\right)+0.9 \cos (t)
\end{array}\right] \\
& d_{2}=\left[\begin{array}{l}
1.5 \dot{q}_{1}+\cos \left(2 q_{1}\right)+2 \cos (t) \\
-0.8 \dot{q}_{2}+0.8 \cos \left(3 q_{2}\right)+1.2 \sin (t) \\
1.5 \dot{q}_{3}-3 \sin \left(3 q_{3}\right)+1.4 \sin (t)
\end{array}\right]
\end{aligned}
$$

where $d_{1}$ represent the disturbances in the signal transformation from joint space to task space, which are mismatched disturbances. $d_{2}$ denote the disturbances when 
Table 2: Controller parameters

\begin{tabular}{llllllll}
\hline Parameters & $\delta$ & $v_{0}$ & $T_{f}$ & $v_{T_{f}}$ & $k_{1}$ & $c_{1}$ & $c_{2}$ \\
\hline Value & 1 & 1 & 0.6 & 0.001 & 10 & 5 & 15 \\
\hline Parameters & $a_{1}$ & $a_{2}$ & $h$ & $\beta$ & $L$ & $J$ & $M$ \\
\hline Value & 2 & 0.7 & 10 & 0.01 & $40 I^{3 \times 3}$ & $5 I^{3 \times 3}$ & $8 I^{3 \times}$ \\
\hline
\end{tabular}

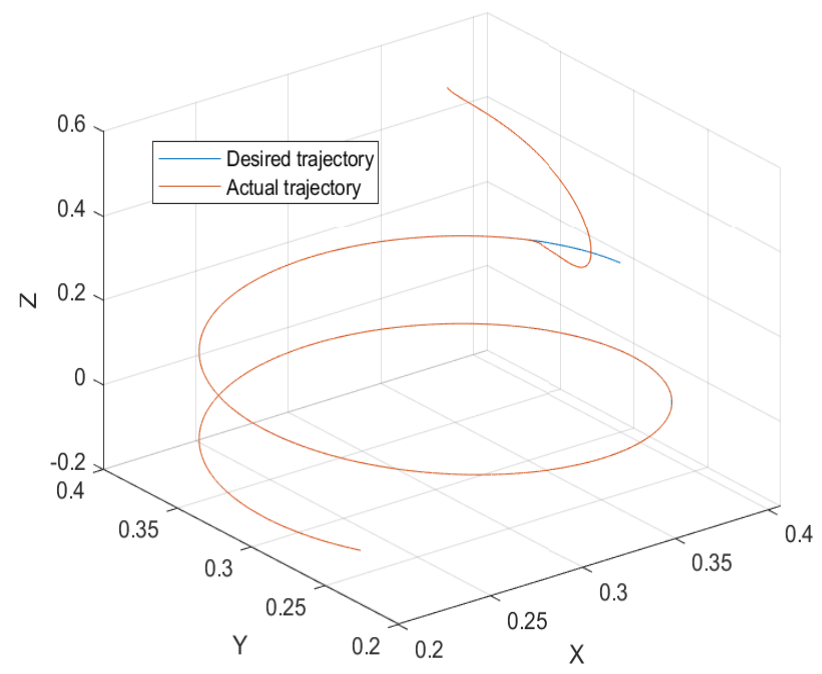

Desired position trajectory and actual position trajectory

the joints are rotated by the torques, which are matched disturbances.

Remark 4: There is no particular reason for the selection of the above disturbance functions, which represent unknown disturbance matrices and can be arbitrarily denoted by a time-varying function with a reasonable magnitude.

To verify the superior performance of the proposed control method in the fast convergence and tracking accuracy, it is compared with other recently proposed advanced control methods, including the adaptive NFTSMC approach indicated in [33], second order NFTSMC strategy presented in [34], adaptive fuzzy integral SMC [18], and adaptive backstepping NFTSMC in [14]. The parameters of our proposed controller are shown in Table 3.

Figure 1 shows the tracking results of the proposed control method for the spatial cylindrical helix. Figure 2 shows the variation of the components of the tracking error in the $x, y, z$ directions in the three-dimensional task space, respectively. It can be seen from the figure 2 that the errors are all restricted to the prescribed performance function. From the above results, it can be seen that our proposed approach achieves accurate

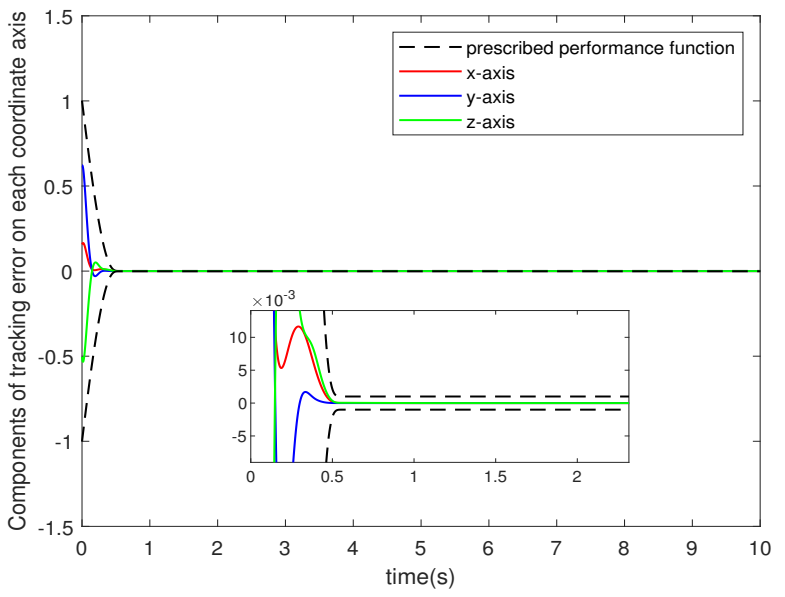

Position trajectory tracking errors

model-free trajectory tracking for the robotic arm system in the presence of matched and mismatched disturbances. And the transient control of errors can be achieved by adjusting the parameters of the prescribed performance function.

Furthermore to demonstrate the superior performance of the proposed control method, it is compared with the recently proposed advanced control methods $[33,34,14,18]$. Since none of the above comparison methods consider the mismatched disturbance, the mismatched disturbance $d_{1}$ in the controlled system is not considered in the simulation comparison of the above approaches, while the proposed control method in this paper still operates in the presence of the mismatched disturbance $d_{2}$.

All comparative simulations are performed in the same initial states. Figures 3 to 5 show the position tracking error of each joint for different control methods. It can be seen from the figures that our controller converges the error to zero in a faster time compared to the other control methods. The speed of error convergence for all these methods, including the approach proposed in this paper, is quite significantly affected by parameters such as proportional gain, and generally the larger the gain value, the faster the system converges, but with greater oscillations. In this simulation, the parameters of each method are selected based on trialand-error method or experience to obtain a balance between convergence speed and oscillation. The main advantage of the controller proposed in this paper is that the control error can be directly limited within a reasonable range by the prescribed performance function, which achieves the control of transient performance. As can be seen from the figure, our control method also obtains smaller steady-state errors. For a more intuitive 

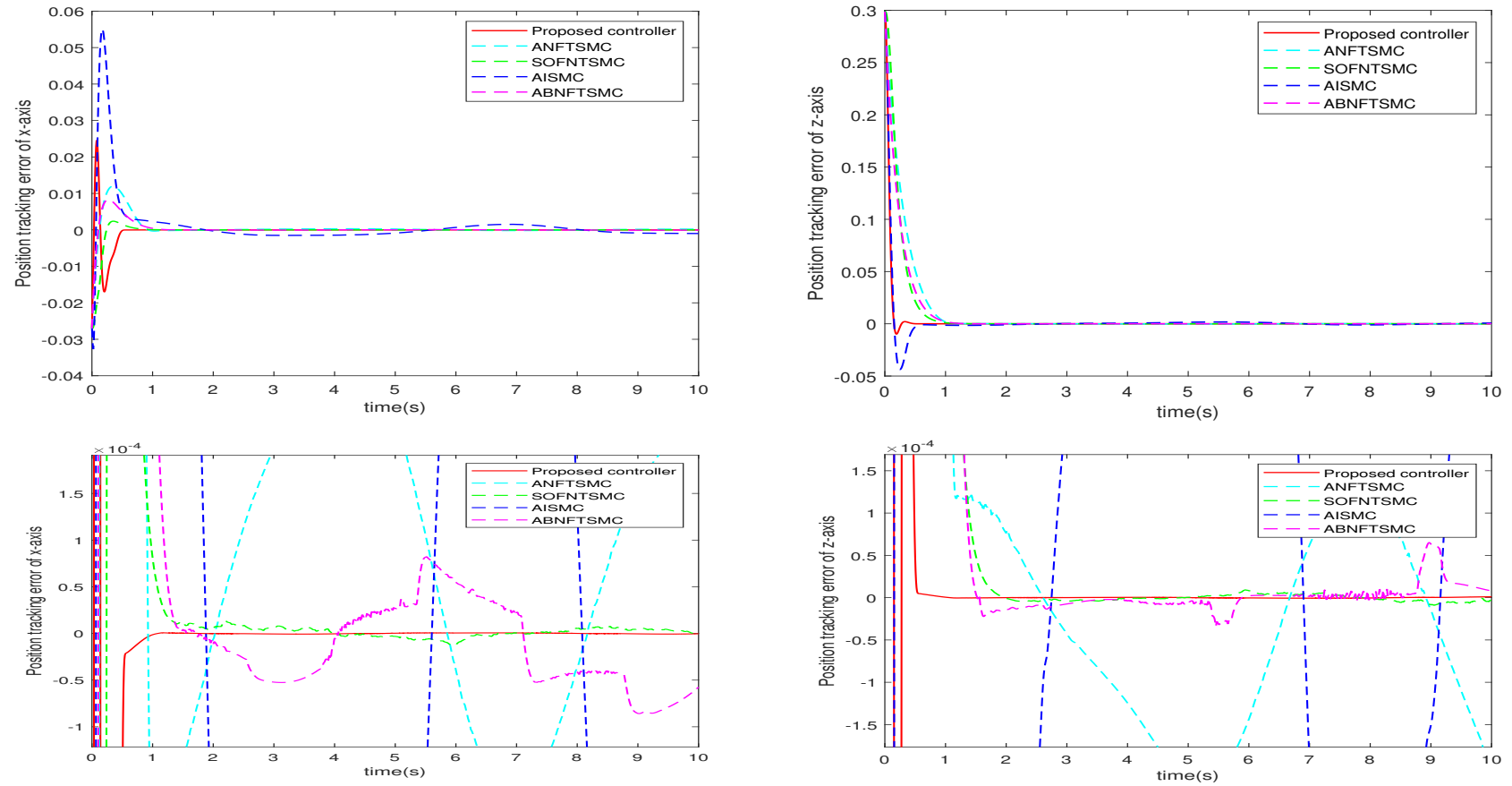

Fig. 3: Tracking error of $\mathrm{X}$-axis

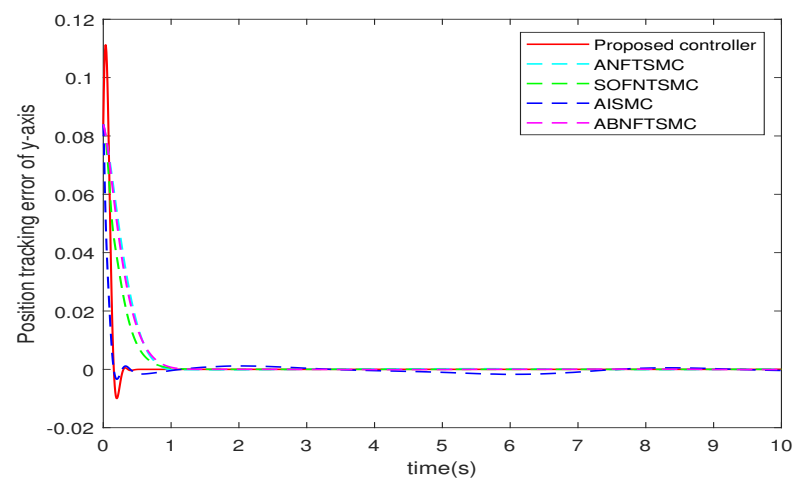

Fig. 5: Tracking error of Z-axis

Table 3: Comparison of the performance indices

\begin{tabular}{|c|c|c|c|}
\hline Controller $A E$ & $A E_{1}$ & $A E_{2}$ & $A E_{3}$ \\
\hline Proposed controller & 0.0005 & 0.0011 & 0.0022 \\
\hline ABNFTSMC & 0.0005 & 0.0025 & 0.0066 \\
\hline ANFTSMC & 0.0008 & 0.0027 & 0.0084 \\
\hline SOFNTSMC & 0.0004 & 0.0020 & 0.0070 \\
\hline AISMC & 0.0024 & 0.0012 & 0.0039 \\
\hline
\end{tabular}

illustration, we introduce the average value of the errors (AE) for quantitative analysis and comparison. The AE are defined as follows:

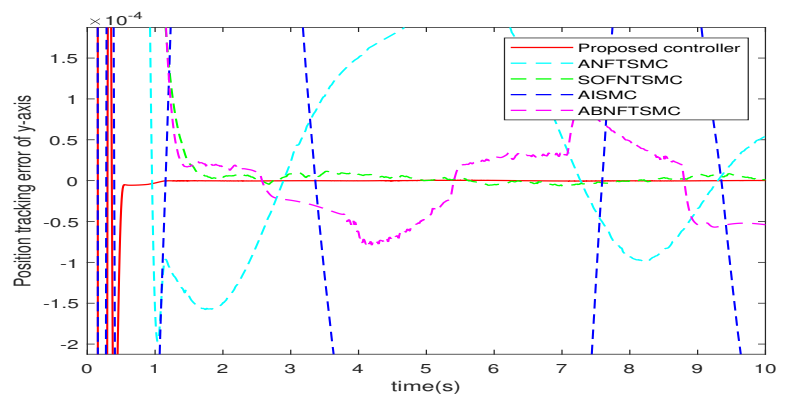

$A E_{j}=\frac{1}{t_{f}-t_{0}} \int_{t_{0}}^{t_{f}}\left|e_{j}(t)\right| d t, \quad j=1,2,3$

where $t_{0}$ and $t_{f}$ are the initial time and the end time, respectively. Performance indicators of the comparative control strategies are reported in Table 3. From the above table, it can be seen that, compared to other methods, even though the controller in this paper operates with the included additional mismatched disturbances, it still obtains the smallest AE values.

Fig. 4: Tracking error of Y-axis

Fig.6 exhibits the Control torque of each joint for different controllers. Because the same desired trajectory is tracked on the same robotic arm model, the torque of the methods used for comparison is basically similar. In contrast, the method proposed in this paper has a large difference in torques from the comparison 

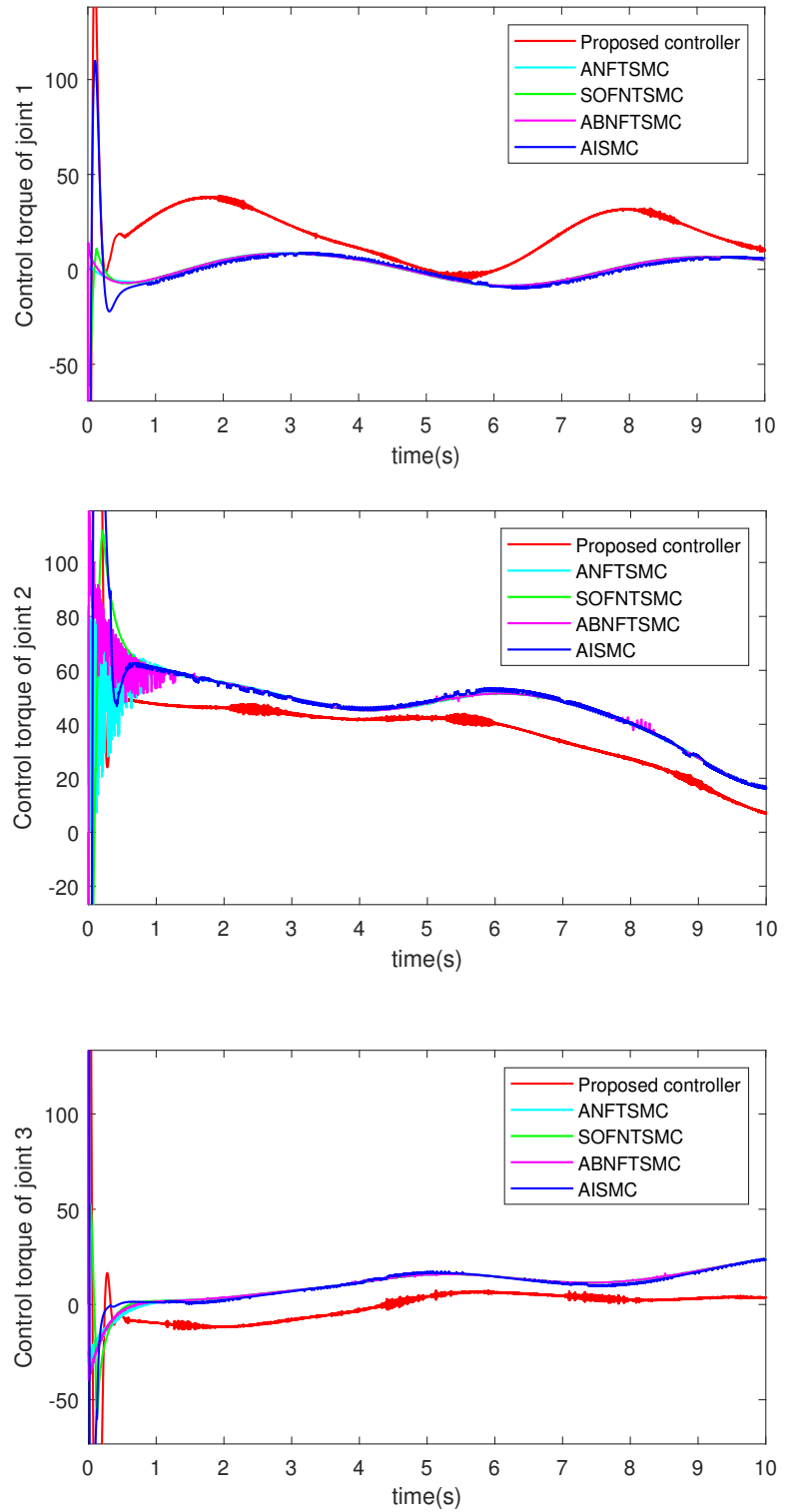

Fig. 6: Control torque of each joint for different control methods

method because of the inclusion of mismatched disturbances. As can be seen from the figure, our controller remains basically smooth, without singularity and with less chatter, even with the additional consideration of mismatched disturbances.

\section{Conclusion}

In this paper, we consider the mismatched uncertainty in the conversion of the robot arm joint space signal to the actual motion of the task space end-effector and design a model-free control method for this problem.
Firstly, the errors are transformed by a prescribed performance function to restrict the errors to a given range to achieve transient control. Then we designed a fast terminal sliding surface for the auxiliary control variables in the backstepping method to improve the convergence speed of the system. The dynamic and kinematic parameters of the robotic arm system are estimated utilizing time delay estimation to achieve modelfree control. Considering the time delay estimation error as part of the external disturbance and designing an observer capable of estimating both matched and mismatched disturbances. The feasibility and superiority of the method in this paper are demonstrated by numerical simulation and theoretical derivation.

In the numerical simulation results, we can find that although we solve mismatched uncertainty, it also triggers the overshoot phenomenon. How to avoid overshoot on the basis of the method proposed in this paper is the focus of our future research.

\section{Declarations}

\section{Funding}

This work is supported by the National Natural Science Foundation of China $(51765042,61963062)$.

\section{Conflicts of interest}

Authors have no conflict of interest to declare.

\section{Data availability}

The data that support the findings of this study are available from the corresponding author upon reasonable request.

\section{References}

1. Pablo Padilla-Iserte, Víctor Lago, Carmen Tauste, Berta Díaz-Feijoo, Antonio Gil-Moreno, Reyes Oliver, Pluvio Coronado, María Belén Martín-Salamanca, Manuel Pantoja-Garrido, Josefina Marcos-Sanmartin, et al. Impact of uterine manipulator on oncological outcome in endometrial cancer surgery. American Journal of Obstetrics and Gynecology, 224(1):65-e1, 2021.

2. Mingxue Cai, Yu Wang, Shuo Wang, Rui Wang, Yong Ren, and Min Tan. Grasping marine products with hybrid-driven underwater vehicle-manipulator system. IEEE Transactions On Automation Science And Engineering, 17(3):1443-1454, 2020. 
3. Dandan Zhang, Jindong Liu, Lin Zhang, and GuangZhong Yang. Hamlyn crm: a compact master manipulator for surgical robot remote control. International journal of computer assisted radiology and surgery, 15(3):503-514, 2020.

4. Angeleene S Ang, Alina Karabchevsky, Igor V Minin, Oleg V Minin, Sergey V Sukhov, and Alexander S Shalin. 'photonic hook'based optomechanical nanoparticle manipulator. Scientific reports, 8(1):1-7, 2018.

5. Miguel Ángel Trujillo, José Ramiro Martínez-de Dios, Carlos Martín, Antidio Viguria, and Aníbal Ollero. Novel aerial manipulator for accurate and robust industrial ndt contact inspection: A new tool for the oil and gas inspection industry. Sensors, 19(6):1305, 2019.

6. Xin Jin, Kaikang Chen, Yang Zhao, Jiangtao Ji, and Pang Jing. Simulation of hydraulic transplanting robot control system based on fuzzy pid controller. Measurement, 164:108023, 2020.

7. L Angel and J Viola. Fractional order pid for tracking control of a parallel robotic manipulator type delta. ISA transactions, 79:172-188, 2018.

8. Mert Önkol and Coşku Kasnakoğlu. Adaptive model predictive control of a two-wheeled robot manipulator with varying mass. Measurement and Control, 51(1-2):38-56, 2018.

9. Li Dai, Yuantao Yu, Di-Hua Zhai, Teng Huang, and Yuanqing Xia. Robust model predictive tracking control for robot manipulators with disturbances. IEEE Transactions on Industrial Electronics, 2020.

10. Yaoyao Wang, Kangwu Zhu, Bai Chen, and Maolin Jin. Model-free continuous nonsingular fast terminal sliding mode control for cable-driven manipulators. ISA transactions, 98:483-495, 2020.

11. Mehran Rahmani, Hossein Komijani, and Mohammad Habibur Rahman. New sliding mode control of 2dof robot manipulator based on extended grey wolf optimizer. International Journal of Control, Automation and Systems, pages 1-9, 2020.

12. Syed Ali Ajwad, Jamshed Iqbal, Raza Ul Islam, Ahmed Alsheikhy, Abdullah Almeshal, and Adeel Mehmood. Optimal and robust control of multi dof robotic manipulator: Design and hardware realization. Cybernetics and Systems, 49(1):77-93, 2018.

13. Ni Bu, Jinxin Pang, and Mingcong Deng. Robust fault tolerant tracking control for the multi-joint manipulator based on operator theory. Journal of the Franklin Institute, 357(5):2696-2714, 2020

14. Mien Van, Michalis Mavrovouniotis, and Shuzhi Sam Ge. An adaptive backstepping nonsingular fast terminal sliding mode control for robust fault tolerant control of robot manipulators. IEEE Transactions on Systems, Man, and Cybernetics: Systems, 49(7):1448-1458, 2018.

15. Tong Yang, Ning Sun, Yongchun Fang, Xin Xin, and He Chen. New adaptive control methods for n-link robot manipulators with online gravity compensation: Design and experiments. IEEE Transactions on Industrial Electronics, 2021.

16. Saim Ahmed, Haoping Wang, and Yang Tian. Adaptive fractional high-order terminal sliding mode control for nonlinear robotic manipulator under alternating loads. Asian Journal of Control, 2020.

17. Qiong Liu, Dongyu Li, Shuzhi Sam Ge, Ruihang Ji, Zhong Ouyang, and Keng Peng Tee. Adaptive bias rbf neural network control for a robotic manipulator. Neurocomputing, 447:213-223, 2021.

18. Mien Van and Shuzhi Sam Ge. Adaptive fuzzy integral sliding mode control for robust fault tolerant control of robot manipulators with disturbance observer. IEEE Transactions on Fuzzy Systems, 2020.

19. Vu Thi Yen, Wang Yao Nan, and Pham Van Cuong. Recurrent fuzzy wavelet neural networks based on robust adaptive sliding mode control for industrial robot manipulators. Neural Computing and Applications, 31(11):69456958, 2019.

20. Changyin Sun, Hejia Gao, Wei He, and Yao Yu. Fuzzy neural network control of a flexible robotic manipulator using assumed mode method. IEEE transactions on neural networks and learning systems, 29(11):5214-5227, 2018.

21. Alireza Mohammadi, Mahdi Tavakoli, Horacio J Marquez, and Farzad Hashemzadeh. Nonlinear disturbance observer design for robotic manipulators. Control Engineering Practice, 21(3):253-267, 2013.

22. Zhijia Zhao, Xiuyu He, and Choon Ki Ahn. Boundary disturbance observer-based control of a vibrating singlelink flexible manipulator. IEEE Transactions on Systems, Man, and Cybernetics: Systems, 2019.

23. Ling Zhao, Qi Li, Bo Liu, and Haiyan Cheng. Trajectory tracking control of a one degree of freedom manipulator based on a switched sliding mode controller with a novel extended state observer framework. IEEE Transactions on Systems, Man, and Cybernetics: Systems, 49(6):1110-1118, 2017.

24. Jiaping Qiang, Le Liu, Miao Xu, and Yiming Fang. Fixed-time backstepping control based on adaptive super-twisting disturbance observers for a class of nonlinear systems. International Journal of Control, pages 1-13, 2021.

25. Yukai Zhu, Jianzhong Qiao, and Lei Guo. Adaptive sliding mode disturbance observer-based composite control with prescribed performance of space manipulators for target capturing. IEEE Transactions on Industrial Electronics, 66(3):1973-1983, 2018.

26. Tie Zhang and Aimin Zhang. Robust finite-time tracking control for robotic manipulators with time delay estimation. Mathematics, 8(2):165, 2020.

27. Xin Zhang, Jinguo Liu, Qing Gao, and Zhaojie Ju. Adaptive robust decoupling control of multi-arm space robots using time-delay estimation technique. Nonlinear Dynamics, 100:2449-2467, 2020.

28. Hongyan Yang and Shen Yin. Actuator and sensor fault estimation for time-delay markov jump systems with application to wheeled mobile manipulators. IEEE Transactions on Industrial Informatics, 16(5):3222-3232, 2019.

29. Ding Zhai, Liwei An, Jiuxiang Dong, and Qingling Zhang. Robust adaptive fuzzy control of a class of uncertain nonlinear systems with unstable dynamics and mismatched disturbances. IEEE transactions on cybernetics, 48(11):3105-3115, 2017.

30. Wan Min and Qingyou Liu. An improved adaptive fuzzy backstepping control for nonlinear mechanical systems with mismatched uncertainties. Automatika, 60(1):1-10, 2019.

31. Chenghu Jing, Hongguang $\mathrm{Xu}$, and Xinjian Niu. Adaptive sliding mode disturbance rejection control with prescribed performance for robotic manipulators. ISA transactions, 91:41-51, 2019.

32. Min Wang and Anle Yang. Dynamic learning from adaptive neural control of robot manipulators with prescribed performance. IEEE Transactions on Systems, Man, and Cybernetics: Systems, 47(8):2244-2255, 2017.

33. Mohamed Boukattaya, Neila Mezghani, and Tarak Damak. Adaptive nonsingular fast terminal sliding-mode control for the tracking problem of uncertain dynamical systems. Isa Transactions, 77:1-19, 2018. 
34. Shanchao Yi and Junyong Zhai. Adaptive second-order fast nonsingular terminal sliding mode control for robotic manipulators. ISA transactions, 90:41-51, 2019.

35. Brahim Brahmi, Mark Driscoll, Mohamed Hamza Laraki, and Abdelkrim Brahmi. Adaptive high-order sliding mode control based on quasi-time delay estimation for uncertain robot manipulator. Control Theory and Technology, 18(3):279-292, 2020.

36. Pegah Ghaf-Ghanbari, Mahmood Mazare, and Mostafa Taghizadeh. Active fault-tolerant control of a schonflies parallel manipulator based on time delay estimation. Robotica, pages 1-18, 2021.

37. Yaoyao Wang, Jiawei Peng, Kangwu Zhu, Bai Chen, and Hongtao Wu. Adaptive pid-fractional-order nonsingular terminal sliding mode control for cable-driven manipulators using time-delay estimation. International Journal of Systems Science, 51(15):3118-3133, 2020.

38. Guermouche Mohamed, Ahmed Ali Sofiane, and Langlois Nicolas. Adaptive super twisting extended state observer based sliding mode control for diesel engine air path subject to matched and unmatched disturbance. Mathematics and Computers in Simulation, 151:111-130, 2018.

39. Man Zhihong, Andrew P Paplinski, and Hong Ren Wu. A robust mimo terminal sliding mode control scheme for rigid robotic manipulators. IEEE transactions on automatic control, 39(12):2464-2469, 1994.

40. Mirosław Galicki. Finite-time trajectory tracking control in a task space of robotic manipulators. Automatica, 67:165-170, 2016.

41. Arie Levant. Robust exact differentiation via sliding mode technique. automatica, 34(3):379-384, 1998.

42. Maolin Jin, Jinoh Lee, Pyung Hun Chang, and Chintae Choi. Practical nonsingular terminal sliding-mode control of robot manipulators for high-accuracy tracking control. IEEE Transactions on Industrial Electronics, 56(9):3593-3601, 2009.

43. Yassine Kali, Maarouf Saad, Khalid Benjelloun, and Charles Khairallah. Super-twisting algorithm with time delay estimation for uncertain robot manipulators. Nonlinear Dynamics, 93(2):557-569, 2018. 